PROCEEDINGS OF THE

AMERICAN MATHEMATICAL SOCIETY

Volume 133, Number 11, Pages 3429-3435

S 0002-9939(05)07858-5

Article electronically published on May 2, 2005

\title{
COUNTABLE DENSE HOMOGENEITY OF DEFINABLE SPACES
}

\author{
MICHAEL HRUŠÁK AND BEATRIZ ZAMORA AVILÉS
}

(Communicated by Alan Dow)

\begin{abstract}
We investigate which definable separable metric spaces are countable dense homogeneous ( $\mathrm{CDH})$. We prove that a Borel CDH space is completely metrizable and give a complete list of zero-dimensional Borel $\mathrm{CDH}$ spaces. We also show that for a Borel $X \subseteq 2^{\omega}$ the following are equivalent: (1) $X$ is $G_{\delta}$ in $2^{\omega},(2) X^{\omega}$ is $\mathrm{CDH}$ and (3) $X^{\omega}$ is homeomorphic to $2^{\omega}$ or to $\omega^{\omega}$. Assuming the Axiom of Projective Determinacy the results extend to all projective sets and under the Axiom of Determinacy to all separable metric spaces. In particular, modulo a large cardinal assumption it is relatively consistent with $\mathrm{ZF}$ that all $\mathrm{CDH}$ separable metric spaces are completely metrizable. We also answer a question of Steprāns and Zhou, by showing that $\mathfrak{p}=\min \left\{\kappa: 2^{\kappa}\right.$ is not $\left.\mathrm{CDH}\right\}$.
\end{abstract}

\section{INTRODUCTION}

A separable topological space $X$ is countable dense homogeneous $(C D H)$ if given any two countable dense subsets $D, D^{\prime} \subseteq X$ there is a homeomorphism $h$ of $X$ such that $h[D]=D^{\prime}$. The first result in this area is due to Cantor, who, in effect, showed that the reals are CDH. Fréchet $[\mathrm{Fr}]$ and Brouwer $[\mathrm{Br}]$, independently, proved that the same is true for the $n$-dimensional Euclidean space $\mathbb{R}^{n}$. In 1962, Fort [Fo] proved that the Hilbert cube is also CDH.

Systematic study of CDH spaces was initiated by Bennett $\mathrm{Be}$ in 1972. Since then, a number of papers were published on the topic, most of which are mentioned in the references. The focus remained on separable metric spaces. Under some set-theoretic assumptions such as the Continuum Hypothesis or Martin's Axiom, a variety of examples of countable dense homogeneous metric spaces were constructed: assuming $\mathrm{CH}$, Fitzpatrick and Zhou constructed a CDH Bernstein subset of $\mathbb{R}^{n}$ and a $\mathrm{CDH}$ subset of $\mathbb{R}$ which is meager in itself; Baldwin and Beaudoin constructed a Bernstein subset of $\mathbb{R}$ under Martin's Axiom for countable partial orders.

In this paper, we are concerned mostly with the countable dense homogeneity of definable separable metric spaces. Our principal result states that every analytic $\mathrm{CDH}$ space is completely Baire. We use it to give a complete list of zero-dimensional

Received by the editors June 13, 2003 and, in revised form, June 11, 2004

2000 Mathematics Subject Classification. Primary 54E52, 54H05, 03E15.

Key words and phrases. Countable dense homogeneous, Borel, Baire.

The first author's research was supported partially by grant GAČR 201/03/0933 and by a PAPIIT grant IN108802-2 and CONACYT grant 40057-F.

(C)2005 American Mathematical Society Reverts to public domain 28 years from publication 
Borel CDH spaces and to show that for a Borel $X \subseteq 2^{\omega}$ the following are equivalent: (1) $X$ is $G_{\delta}$ in $2^{\omega},(2) X^{\omega}$ is $\mathrm{CDH}$ and (3) $X^{\omega}$ is homeomorphic to $2^{\omega}$ or to $\omega^{\omega}$. These provide partial answers to the following problems of [FZ3]:

387. For which 0-dimensional subsets of $\mathbb{R}$ is $X^{\omega}$ homogeneous? CDH? and

389. Does there exist a CDH metric space that is not completely metrizable?

\section{Descriptive Set Theory}

In this section, we review some of the classical results of descriptive set theory. For proofs and further references consult e.g. [Ke]. Recall that a separable completely metrizable space is called a Polish space. We call a separable metric space Borel if it is Borel in its completion. A separable metric space is analytic if it is a continuous image of the Baire space $\omega^{\omega}$. A space is co-analytic if it is a complement of an analytic subspace of some Polish space. Recall that a space is Borel if and only if it is both analytic and co-analytic. This is an old result of Souslin as is the following:

Theorem 1.1. Every uncountable analytic space contains a homeomorphic copy of $2^{\omega}$.

Recall that a subset $A$ of a Polish space $X$ is said to have the Baire property if there is an open set $U \subseteq X$ such that the symmetric difference $A \triangle U$ is meager in $X$.

Theorem 1.2. Every analytic subspace of a Polish space has the Baire property.

A topological space $X$ is Baire if the complement of every meager subset of $X$ is dense in $X$. Note that being Baire and having the Baire property are quite different notions. We will use the following corollary of Theorem 1.2 proved in a more general context by Levi [Le] (see also VM2]):

Theorem 1.3. Every analytic Baire space has a dense completely metrizable subspace.

Proof. Let $X$ be an analytic Baire space and let $\bar{X}$ be its completion. By Theorem 1.2 , there is an open set $U \subseteq \bar{X}$ such that $X \triangle U$ is meager in $\bar{X}$. That is, $X \triangle U=$ $\bigcup_{n \in \omega} F_{n}$, where each $F_{n}$ is nowhere dense in $\bar{X}$. Note that $U$ is a dense open subset of $\bar{X}$. Let $G=U \backslash \bigcup_{n \in \omega} \bar{F}_{n}$. Then $G$ is completely metrizable as it is $G_{\delta}$ in $\bar{X}$, and $G$ is a dense subset of $X$ as $X$ is Baire.

A topological space $X$ is completely Baire if all of its closed subspaces are Baire. The following theorem is due to Hurewicz (see [Ke]).

Theorem 1.4. Every co-analytic completely Baire space is completely metrizable.

Under the Axiom of Projective Determinacy (PD) all of the above theorems hold for all projective sets. Similarly under the Axiom of Determinacy (AD) they hold for all separable metric spaces. For proof of the analogues of Theorems 1.1 and 1.2 (and hence also Theorem 1.3) in this context see e.g Theorem 27.9 of $\mathrm{Ka}$. The fact that the variants of Theorem 1.4 hold follows from the proof of Theorem 4 of KLW.

The following characterization of zero-dimensional Polish spaces can be found in [Ke] and [vM2]. 
Theorem 1.5. (i) Every zero-dimensional separable compact completely metrizable space without isolated points is homeomorphic to $2^{\omega}$.

(ii) Every zero-dimensional separable locally compact non-compact completely metrizable space without isolated points is homeomorphic to $2^{\omega} \backslash\{0\}$.

(iii) Every zero-dimensional separable completely metrizable space without isolated points in which all compact sets are nowhere dense is homeomorphic to $\omega^{\omega}$.

\section{Analytic CDH SPACES}

In the article Some open problems in densely homogeneous spaces of Open Problems in Topology, Fitzpatrick and Zhou ask (Question 389) whether there is a CDH metric space which is not completely metrizable. We answer this question in the negative for Borel spaces. The following simple lemma ([FZ2]) will be used many times in what follows.

Lemma 2.1. A separable metric space $X$ without isolated points is meager in itself if and only if there is a countable dense $D \subseteq X$ which is $G_{\delta}$ in $X$.

Proof. The reverse implication is obvious. For the forward implication, let $X=$ $\bigcup_{n \in \omega} F_{n}$, where each $F_{n}$ is a closed nowhere dense subset of $X$. Enumerate a basis for the topology of $X$ as $\left\{U_{n}: n \in \omega\right\}$ and recursively pick $x_{n} \in U_{n} \backslash \bigcup_{m \leq n} F_{m}$. Set $D=\left\{x_{n}: n \in \omega\right\}$. $D$ is obviously a countable dense subset of $X$. To see that it is $G_{\delta}$ in $X$ note that $D$ intersects each $F_{n}$ in a finite set; hence $X \backslash D=\bigcup_{n \in \omega}\left(F_{n} \backslash D\right)$ is $F_{\sigma}$ in $X$.

Next we prove a decomposition lemma for $\mathrm{CDH}$ spaces.

Lemma 2.2. Every $C D H$ space $X$ can be written as a disjoint topological sum $X=I \oplus L \oplus R$, where $I$ is the set of isolated points in $X, L$ is locally compact without isolated points and $R$ has the property that every compact subset or $R$ is nowhere dense in $R$.

Proof. First we show that the set $I$ of all isolated points of $X$ is clopen in $X$. Note that $I$ is countable as $X$ is separable. If $I$ is not closed, pick $x \in \bar{I} \backslash I$ and a set $C \subseteq X \backslash \bar{I}$ countable dense in $X \backslash \bar{I}$. Let $D_{0}=I \cup C$ and $D_{1}=$ $D_{0} \cup\{x\}$. The sets $D_{0}$ and $D_{1}$ are then countable dense subsets of $X$, and we reach a contradiction by noting that there is no homeomorphism of $X$ sending $D_{1}$ to $D_{0}$, for $x$ is not isolated but every neighborhood of $x$ contains an isolated point, whereas all points in $D_{0}$ are either isolated or have a neighborhood which does not contain any isolated points. Let $Y=X \backslash I$. Consider $L=\{x \in Y: \exists U \subseteq Y$ a locally compact neighborhood of $x\}$ and $R=\{x \in Y$ : $\exists U \subseteq Y$ a neighborhood of $x$, s.t. $\forall K \subseteq U$ compact, $\operatorname{int}(K)=\emptyset\}$. Obviously, $L$ and $R$ are disjoint open subsets of $Y$. To finish the proof it suffices to show that $Y=L \cup R$. First note that $L \cup R$ is dense in $Y$, as if $x \in Y \backslash(L \cup R)$ then i.p. $x \in Y \backslash R$, which implies that for every $U \subseteq X$ neighborhood of $x$, there is a $K \subseteq U$ compact such that $\operatorname{int}(K) \neq \emptyset$; hence $x \in \bar{L}$.

Now, suppose that $Y \backslash(L \cup R) \neq \emptyset$. Pick $x \in Y \backslash(L \cup R)$ and a countable dense $D_{0} \subseteq L \cup R$ and let $D_{1}=D_{0} \cup\{x\}$. Again, $D_{0}$ and $D_{1}$ are clearly countable in $X$ and there is no homeomorphism $h$ of $X$ sending $D_{1}$ to $D_{0}$ as then $h(x) \in L$ or $h(x) \in R$ but $x \notin L \cup R$.

Theorem 2.3. Every analytic $C D H$ space $X$ is completely Baire. 
Proof. By Lemma 2.2, we can assume that $X$ has no isolated points.

Claim 1. Every open subset of $X$ is uncountable.

Assume not, that is, $V=\bigcup\{U: U$ is a countable open subset of $X\}$ is not empty. Then $V$ is itself a countable open set. Choose $C$ a countable dense subset of $X \backslash V$ and $x \in V$. Let $D_{0}=C \cup V$ and $D_{1}=C \cup V \backslash\{x\}$. The sets $D_{0}$ and $D_{1}$ are then countable dense subsets of $X$. As $X$ is CDH there is a homeomorphism $h$ of $X$ such that $h\left[D_{1}\right]=D_{0}$. Then, however, $h(x) \notin V$ and, unlike $x, h(x)$ does not have a countable neighborhood, which contradicts the fact that $h$ is a homeomorphism.

Claim 2. $X$ is Baire.

Suppose it is not the case. That means that there is an open set $U \subseteq X$ which is meager in itself. By Lemma 2.1, there is a $C \subseteq U$ countable dense in $U$ which is $G_{\delta}$ in $U$. Let $D_{0}$ be a countable dense subset of $X$ such that $D_{0} \cap U=C$.

Let $\left\{U_{n}: n \in \omega\right\}$ be an enumeration of some countable basis for the topology on $X$. By Claim 1, each $U_{n}$ is uncountable, and as every open subset of an analytic space is itself analytic, by Theorem 1.1, each $U_{n}$ contains a subset $F_{n}$ homeomorphic to $2^{\omega}$. Choose, for every $n \in \omega$, a countable $C_{n} \subseteq F_{n}$ dense in $F_{n}$ and set $D_{1}=$ $\bigcup_{n \in \omega} C_{n}$. The set $D_{1}$ is then a countable dense subset of $X$.

Note that $D_{1} \cap V$ is not $G_{\delta}$ in $V$ for any open set $V \subseteq X$. To see this, let $V$ be an open subset of $X$. There is an $n \in \omega$ such that $U_{n} \subseteq V$; hence $F_{n} \subseteq V$. If $D_{1} \cap V$ were $G_{\delta}$ in $V$, then $D_{1} \cap F_{n}$ would be $G_{\delta}$ in $F_{n}$. As $C_{n} \subseteq D_{1} \cap F_{n}$ it follows that $D_{1} \cap F_{n}$ is dense in $F_{n}$. Lemma 2.1 then implies that $F_{n}$ is meager in itself, which contradicts the Baire Category Theorem for $2^{\omega}$.

To finish the proof of the claim it suffices to notice that the countable dense sets $D_{0}$ and $D_{1}$ have different (relative) topological properties in $X$. Hence there is no homeomorphism of $X$ sending one to the other, which contradicts the fact that $X$ is $\mathrm{CDH}$.

We are now ready to show that $X$ is completely Baire. By Claim 2 and Theorem 1.3 , there is a completely metrizable $G \subseteq X$ which is dense in $X$. Let $D_{0}$ be any countable dense subset of $G$ (and consequently also a dense subset of $X$ ). Note that $D_{0}$ has the property that if $E \subseteq D_{0}$ has no isolated points, then $E$ is not $G_{\delta}$ in $\bar{E}$, for if $E$ were $G_{\delta}$ in $\bar{E}$, then $E$ would be $G_{\delta}$ in $\bar{E} \cap G$, but $\bar{E} \cap G$ is a $G_{\delta}$ subset of $G$, hence, is completely metrizable. However, by the Baire Category Theorem this does not happen.

Aiming toward a contradiction again, assume that $X$ is not completely Baire. That is, there is a closed set $F \subseteq X$ which is meager in itself. By Lemma 2.1, there is a countable dense $C \subseteq F$ which is $G_{\delta}$ in $F$. Let $D_{1}=C \cup\left(D_{0} \backslash F\right)$. The set $D_{1}$ is clearly a countable dense subset of $X$ and has the property that there is a subset of it without isolated points which is $G_{\delta}$ in its closure ( $C$ being a witness to this).

So, again, the countable dense sets $D_{0}$ and $D_{1}$ have different (relative) topological properties in $X$. Hence there is no homeomorphism of $X$ sending one to the other, contradicting the countable dense homogeneity of $X$.

Corollary 2.4. Every Borel CDH space $X$ is completely metrizable.

Proof. This follows directly from Theorem 2.3 and Theorem 1.4.

Corollary 2.5. Let $X$ be a zero-dimensional Borel CDH space without isolated points. Then $X$ is homeomorphic to one of the following five spaces: $2^{\omega}$, $\omega^{\omega}$, $2^{\omega} \backslash\{0\}, \omega^{\omega} \oplus 2^{\omega}$ or $\omega^{\omega} \oplus 2^{\omega} \backslash\{0\}$. 
Proof. By the previous corollary, $X$ is completely metrizable. By Lemma 2.2, $X=L \oplus R$, where $L$ is locally compact without isolated points and $R$ has the property that every compact subset of $R$ is nowhere dense in $R$. By Theorem 1.5, $R$ is either empty or homeomorphic to $\omega^{\omega}$ and $L$ (if non-empty) is homeomorphic either to $2^{\omega}$ or $2^{\omega} \backslash\{0\}$, depending on whether it is compact or not.

A natural question is whether the above results can be extended beyond analytic or Borel sets. The answer depends on set-theoretic assumptions. For possible extensions, note that all arguments presented so far use only the validity of Theorems 1.1, 1.3, 1.4 and only the countable Axiom of Choice, a consequence of the Axiom of Dependent Choice.

Corollary 2.7. (i) (PD) Every projective CDH space is completely metrizable.

(ii) (AD) All separable metric CDH spaces are completely metrizable.

So in particular, it is consistent with ZF that every zero-dimensional metric $\mathrm{CDH}$ space without an isolated point is homeomorphic to one of the following spaces: $2^{\omega}$, $\omega^{\omega}, 2^{\omega} \backslash\{0\}, \omega^{\omega} \oplus 2^{\omega}$ or $\omega^{\omega} \oplus 2^{\omega} \backslash\{0\}$.

To conclude the section we show that Theorem 2.3 and Corollary 2.4 are consistently sharp by proving the following:

Theorem 2.6. $\left(\mathrm{MA}+\neg \mathrm{CH}+\omega_{1}=\omega_{1}^{L}\right)$ Let $X$ be an $\aleph_{1}$-dense subset of $2^{\omega}$. Then:

(i) $X$ is a co-analytic meager in itself $C D H$ space.

(ii) $2^{\omega} \backslash X$ is an analytic completely Baire CDH space which is not completely metrizable.

Proof. A theorem of Martin and Solovay (see [Mi]) states that, assuming MA + $\neg \mathrm{CH}+\omega_{1}=\omega_{1}^{L}$, every set of reals of size $\aleph_{1}$ is co-analytic. MA implies that $X$ is meager in itself. It is easy to see that $2^{\omega} \backslash X$ is completely Baire and not completely metrizable (and of course analytic).

The fact that both $X$ and $2^{\omega} \backslash X$ are CDH follows directly from Lemma 3.1 of [BB].

\section{Products of CDH spaces}

Products of $\mathrm{CDH}$ spaces need not be $\mathrm{CDH}$. A simple example, pointed out by the referee, is the pair $\mathbb{R}$ and $\omega^{\omega}$. On the other hand, infinite products of spaces which are not $\mathrm{CDH}$ can be $\mathrm{CDH}$, an example being the Hilbert cube $[0,1]^{\omega}([\mathrm{Fo}]$ ). Lawrence [La showed that $X^{\omega}$ is homogeneous, for every $X \subseteq 2^{\omega}$ (see also [DP]) answering half of Question 388 of ZH3. The other half asks for which $X \subseteq 2^{\omega}$ is $X^{\omega}$ CDH. It was known that not for all $X$ as Fitzpatrick and Zhou in [FZ2] showed that $\mathbb{Q}^{\omega}$ is not $\mathrm{CDH}$, where $\mathbb{Q}$ denotes the space of rational numbers. In this section, we characterize those Borel subsets of $2^{\omega}$ whose power is $\mathrm{CDH}$.

Theorem 3.1. Let $X$ be a separable metric space such that $X^{\omega}$ is $C D H$. Then $X$ is a Baire space.

Proof. The proof of this theorem is quite analogous to the proof of Claim 2 of Theorem 2.3. Suppose that $X$ has at least two elements. It suffices to note that (1) if $X$ is not Baire, then $X^{\omega}$ is meager in itself, and (2) every open subset of $X^{\omega}$ contains a copy of $2^{\omega}$. 
Theorem 3.2. Let $X \subseteq 2^{\omega}$ be Borel. Then the following are equivalent:

(1) $X^{\omega}$ is $C D H$,

(2) $X$ is $G_{\delta}$ in $2^{\omega}$,

(3) $\left|X^{\omega}\right|=1$ or $X^{\omega}$ is homeomorphic to $2^{\omega}$ or $X^{\omega}$ is homeomorphic to $\omega^{\omega}$.

Proof. Suppose that $\left|X^{\omega}\right|>1$. (1) implies (2) by Theorem 2.3 as $X^{\omega}$ is Borel if and only if $X$ is and, moreover, $X^{\omega}$ is completely metrizable if and only if $X$ is.

To see that (2) implies (3), note that if $X$ is $G_{\delta}$ in $2^{\omega}$, then $X^{\omega}$ is completely metrizable. Moreover, if $X$ is zero-dimensional, then so is $X^{\omega}$ and $X^{\omega}$ does not contain any isolated points. Now, if $X$ is compact, then so is $X^{\omega}$; hence, $X^{\omega}$ is homeomorphic to $2^{\omega}$ by Theorem 1.5 (i). If $X$ is not compact, then all compact subsets of $X^{\omega}$ are nowhere dense and $X^{\omega}$ is homeomorphic to $\omega^{\omega}$ by Theorem 1.5 (ii).

(3) implies (1), as both $2^{\omega}$ and $\omega^{\omega}$ are $\mathrm{CDH}$.

Just like in the previous section this theorem can be strengthened assuming PD or $\mathrm{AD}$. The following question, however, remains open.

Question 3.2. Is there a non-G $G_{\delta}$ subset of $2^{\omega}$ such that $X^{\omega}$ is $C D H$ ?

We will conclude this section and the paper by considering uncountable products. Recall that a family $\mathcal{F} \subseteq[\omega]^{\omega}$ is centered if every non-empty finite subfamily of $\mathcal{F}$ has an infinite intersection. An infinite set $A \subseteq \omega$ is a pseudo-intersection of a family $\mathcal{F} \subseteq[\omega]^{\omega}$ if $A \backslash F$ is finite for every $F \in \mathcal{F}$. The cardinal invariant $\mathfrak{p}$ is defined as the minimal cardinality of a centered family $\mathcal{F} \subseteq[\omega]^{\omega}$ which has no infinite pseudo-intersection.

Steprāns and Zhou in [SZ] showed that $2^{\kappa}$ is CDH for every $\kappa<\mathfrak{p}$ and asked whether $2^{\mathfrak{p}}$ is provably not $\mathrm{CDH}$. We show that it follows from known results that the answer is positive.

Theorem 3.3. $\mathfrak{p}=\min \left\{\kappa: 2^{\kappa}\right.$ is not $\left.C D H\right\}$.

Proof. The fact that $\min \left\{\kappa: 2^{\kappa}\right.$ is not $\left.\mathrm{CDH}\right\} \leq \mathfrak{p}$ was proved in [SZ]. In Ma and [HS], it is shown that there is a countable dense set $D \subseteq 2^{\mathfrak{p}}$ and a point $x \in 2^{\mathfrak{p}}$ such that no sequence in $D$ converges to $x$. On the other hand, it is easy to construct a countable dense set $C \subseteq 2^{\mathfrak{p}}$ such that for every $c \in C$ there is a sequence $\left\langle c_{n}: n \in \omega\right\rangle \subseteq C \backslash\{c\}$ converging to $c$.

Now, notice that there is no homeomorphism of $2^{\mathfrak{p}}$ sending $C$ to $D \cup\{x\}$ as if $c=h^{-1}(x)$ and $\left\langle c_{n}: n \in \omega\right\rangle \subseteq C \backslash\{c\}$ is a sequence converging to $c$, then the sequence $\left\langle h\left(c_{n}\right): n \in \omega\right\rangle$ does not converge to $x$, contradicting continuity of $h$.

\section{ACKNOWLEDGMENTS}

The work contained in this paper is part of the second author's Master's thesis at the Universidad Michoacana de San Nicolás de Hidalgo, written under the supervision of the first author. The first author wishes to thank A. Louveau, I. Farah, J. van Mill and S. Todorčević for bibliographical information and fruitful discussion.

\section{REFERENCES}

[BB] S. Baldwin and R. E. Beaudoin, Countable dense homogeneous spaces under Martin's axiom, Israel J. Math. 65 (1989), 153-164. MR0998668 (90f:54010)

[Be] R. B. Bennett, Countable dense homogeneous spaces, Fund. Math 74 (1972), 189-194. MF 0301711 (46:866) 
[Br] L. E. J. Brouwer, Some Remarks on the coherence type $\eta$, Proc. Akad. Amsterdam 15 (1912), 1256-1263.

[DP] A. Dow and E. Pearl, Homogeneity in Powers of zero-dimensional, first-countable spaces, Proc. AMS 125 (1997), 2503-2510. MR1416083 (97j:54008)

[Fi] B. Fitzpatrick Jr., A note on countable dense homogeneity, Fund. Math. 75 (1972), 3-4. MF 0301696 (46:852)

[FL] B. Fitzpatrick, Jr. and N. F. Lauer, Densely homogeneous spaces. I, Houston J. Math. 13 (1987), 19-25. MR0884229 (88d:54041)

[FZ1] B. Fitzpatrick Jr. and H.-X. Zhou, Densely homogeneous spaces II, Houston J. Math. 14 (1988), 57-68. MR0959223|(89k:54080)

[FZ2] B. Fitzpatrick Jr. and H.-X. Zhou, Countable dense homogeneity and the Baire property, Topology and its Applications 43 (1992), 1-14. MR1141367 (93b:54030)

[FZ3] B. Fitzpatrick Jr. and H.-X. Zhou, Some Open Problems in Densely Homogeneous Spaces, in Open Problems in Topology (ed. J. van Mill and M. Reed), 1984, pp. 251-259, North-Holland, Amsterdam. MF 1078651

[Fo] M. Fort, Homogeneity of infinite products of manifolds with boundary, Pacific J. Math 12 (1962), 879-884. MR0145499 (26:3030)

[Fr] M. Fréchet, Les dimensions d'unensemble abstrait, Math. Ann 68 (1910), 145-168.

[HS] M. Hrušák and J. Steprans, Cardinal invariants related to sequential separability, Surikaisekikenkiusho Kokyuroku 1202 (2001), 66-74. MR1855551

[Ka] A. Kanamori, The Higher Infinite, 1994, Springer-Verlag. MR.1321144 (96k:03125)

[Ke] A. S. Kechris, Classical Descriptive Set Theory, 1995, Springer-Verlag. MR1321597 (96e:03057)

[KLW] A. S. Kechris, A. Louveau and W. H. Woodin, The Structure of $\sigma$-ideals of Compact Sets, Trans. AMS 301 (1987), 263-288. MR 0879573 (88f:03042)

[Ku] K. Kunen, Set Theory, An Introduction to Independence Proofs, 1990, North Holland. MF 0756630 (85e:03003)

[La] B. Lawrence, Homogeneity in powers of subspaces of the real line, Trans. AMS 350 (1998), 3055-3064. MR,1458308 (98k:54061)

[Le] S. Levi, On Baire cosmic spaces, Proceednigs of the Fifth Prague Topological Symposium, 1983, pp. 450-451, Heldermann Verlag, Berlin. MR0698438 (84d:54052)

[Ma] M. V. Matveev, Cardinal $\mathfrak{p}$ and a theorem of Pelczynski, (preprint).

[vM1] J. van Mill, Strong local homogeneity does not imply countable dense homogeneity, Proc. AMS 84 (1982), 143-148. MR0633296 (83e:54033)

[vM2] J. van Mill, The Infinite-Dimensional Topology of Function Spaces, 2001, North Holland. MR1851014 (2002h:57031)

[Mi] A. W. Miller, Descriptive Set Theory and Forcing, 1995, Springer, Lecture Notes in Logic 4. MR.1439251 (98g:03119)

[Sa] W. L. Saltsman, Concerning the existence of a connected, countable dense homogeneous subset of the plane which is not strongly locally homogeneous, Topology Proceedings 16 (1991), 137-176. MR1206461 (94c:54008)

[SZ] J. Steprans, H.-X. Zhou, Some Results on CDH Spaces, Topology and its Applications 28 (1988), 147-154. MR0932979 (89c:54070)

[Zh] H.-X. Zhou, Two applications of set theory to homogeneity, Questions Answers Gen. Topology 6 (1988), 49-56. MR0940441 (89c:54010)

Instituto de Matemáticas, Unam, Unidad Morelia, A. P. 61-3, Xangari, C. P. 58089, Morelia, MichoacÁn, MéXiCo

E-mail address: michael@matmor.unam.mx

Instituto de Matemáticas, UnAm, Unidad Morelia, A. P. 61-3, Xangari, C. P. 58089, Morelia, Michoacán, México

E-mail address: bzamora@matmor.unam.mx 